\title{
PILLAR ARRAY MICRO-TRAPS WITH NEGATIVE DIELECTROPHORESIS
}

\author{
H.H. Cui ${ }^{1}$, J. Voldman ${ }^{1,2}$ and K.M. Lim ${ }^{1,3}$ \\ ${ }^{1}$ Singapore-MIT Alliance, National University of Singapore \\ ${ }^{2}$ Research Laboratory of Electronics, MIT, USA \\ ${ }^{3}$ Department of Mechanical Engineering, National University of Singapore
}

\begin{abstract}
We pesent a microfluidic cell-trap that utilizes negative dielectrophoresis (nDEP) force and hydrodynamic force generated by a pillar array capable of controlling the number of trapped particles accurately. By adjusting of the relative strength of the $\mathrm{nDEP}$ and hydrodynamic forces, the trap located at the region close to the stagnation point of pillar functions as both a single-particle trap (capable of discriminating particles based on size or material properties) and a multi-particle trap (capable of controlling the number of particles trapped). We used $5 \mu \mathrm{m}$ PS beads to validate and demonstrate the capability of this new cell-trap design. Pulsed nDEP is used to increase the selectivity and stability. Good correlation between simulation and the experiment results was obtained.
\end{abstract}

KEYWORDS: Cell-Trap, Dielectrophoresis, Pillar array.

\section{INTRODUCTION}

Trapping of single particle in microfluidic devices is very important due to its potential application in single cell analysis. DEP provides a simple and inexpensive way for trapping and manipulating particles by varying the voltage, frequency or phase of the electrical signal applied to the electrodes in such devices. So far, a few research groups have demonstrated the ability to trap a single particle in their devices [1] [2]. However, there are still some drawbacks, such as the ignorance of the particle-particle interaction and the complicated fabrication. In our present work, we developed a novel device in which a pulsed nDEP force, instead of continuous $\mathrm{nDEP}$, is used to achieve a stable single/multi-particle trap.

\section{THEORY AND SIMULATION}

Figure 1 shows a schematic drawing of a representative section in a pillar array structure. The actual array consists of a 20 by 30 grid fabricated in a microchip.

For a spherical particle with radius $r$ suspended in a medium, the DEP force is given by,

$$
F_{D E P}=2 \pi \varepsilon_{0} \varepsilon_{f} r^{3} \operatorname{Re}\left[K^{*}(\omega)\right] \nabla E_{r m s}^{2}
$$

where $E_{r m s}$ is the root mean square of electrical field strength. The direction of DEP depends on the sign of $\operatorname{Re}\left(K^{*}(\omega)\right)$. Countered by the hydrodynamic force, the sphere reaches a terminal velocity $U_{p}$ given by,

$$
U_{p}=\frac{F_{D E P}}{6 \pi r \mu}+U_{f}
$$


where $\mu$ is the viscosity of fluid, and $U_{f}$ the fluid velocity. In this work, a commercial finite element software (COMSOL, Inc.) is used to analyse the steady state electric field and fluid flow field.The region where particles will be trapped is given by that where the $\mathrm{X}$-component of the particle velocity is less than zero (as given by Eq. 2). The region is highlighted by the black contour in Figure 2.

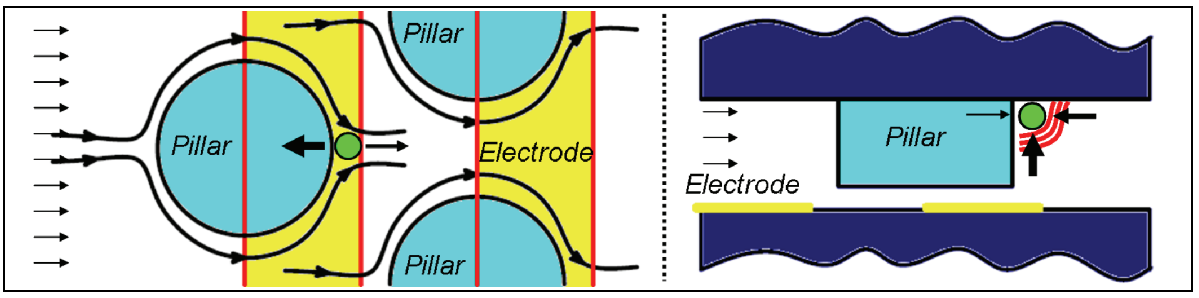

Figure 1. Top view (left panel) and side view (right panel) of the pillar array. The pillar has diameter of $45 \mu \mathrm{m}$ and height of $30 \mu \mathrm{m}$. The distance between the centers of two pillars is $70 \mu \mathrm{m}$. Both the width of electrodes and the gap between are 30 $\mu \mathrm{m}$. The diameter of beads used is $5 \mu \mathrm{m}$. The vertical component of $n D E P$ pushes the beads to top surface and the horizon component of $n D E P$ pushes the beads back to the stagnation point behind the pillar. By adjusting the relative strength of the DEP and hydrodynamic forces, a controllable trap is realized. Pulsing the DEP force makes the trap more stable.

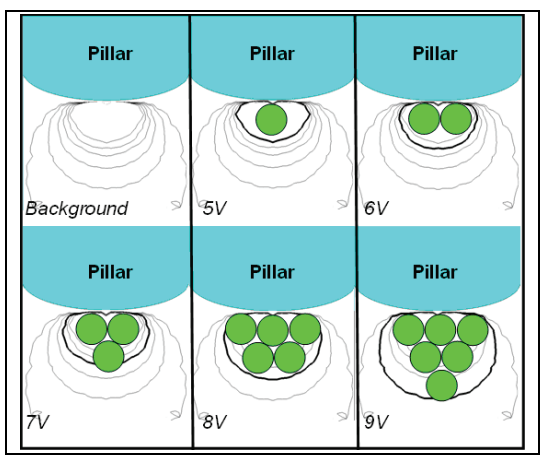

Figure 2. Simulation results to show the patterns of different number of trapped beads under different applied voltages.
Table 1. Experimental data showing the number of trapped beads for various combinations of fluid flow rate and applied voltage.

\begin{tabular}{c|cccccc}
\hline \multirow{2}{*}{$\begin{array}{c}\text { Flow rate } \\
(\mathbf{m L} / \mathbf{h})\end{array}$} & \multicolumn{6}{|c}{ Voltage (Vpp) } \\
\cline { 2 - 7 } & $\mathbf{1 0}$ & $\mathbf{9}$ & $\mathbf{8}$ & $\mathbf{7}$ & $\mathbf{6}$ & $\mathbf{5}$ \\
\hline 0.13 & 1 & 0 & 0 & 0 & 0 & 0 \\
0.12 & 1 & 1 & 0 & 0 & 0 & 0 \\
0.11 & 3 & 2 & 1 & 0 & 0 & 0 \\
0.1 & 5 & 3 & 3 & 1 & 0 & 0 \\
0.09 & 5 & 3 & 3 & 1 & 0 & 0 \\
0.08 & $>5$ & 5 & 3 & 1 & 1 & 0 \\
0.07 & $>5$ & 5 & 3 & 2 & 1 & 1 \\
\hline
\end{tabular}

\section{EXPERIMENTAL METHOD}

ITO coated glass was used because of its high transparency and good electrical conductivity. Standard photolithography is used to pattern the interdigital electrodes (IDEs). Two SU-8 layers were then fabricated: one for pillar array and is the other for the rectangular channel. PS beads were mixed with water and a little surfactant is added to reduce the non-specific adhesion. A $10 \mathrm{MHz}$ sinusoidal voltage up to $20 \mathrm{~V}_{\mathrm{pp}}$ 
generated by signal generator is applied to the electrodes. The built-in amplitude modulating function of the bsignal generator is also used to produce the pulsed DEP.

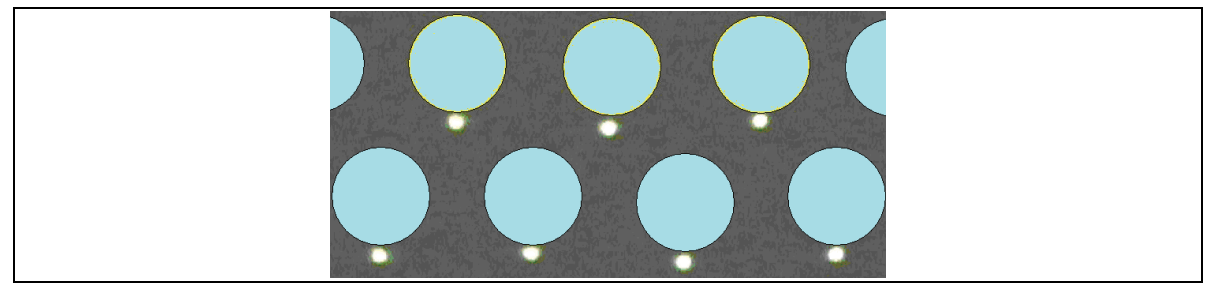

Figure 3. Fluorescent image of experimental result under pulsed nDEP to trap single $5 \mu \mathrm{m}$ bead in the pillar array. Blue circles show the position of the $S U-8$ pillars which are actually invisible under the fluorescent microscope. Small light dots represent the trapped fluorescent $5 \mu \mathrm{m}$ beads. Fluid flows from top to bottom.

\section{RESULTS AND DISCUSSION}

Table 1 shows the number of particles trapped when continuous nDEP was applied, for various combinations of voltage and fluid flow velocity. When pulsed $\mathrm{nDEP}$ is used, the trap was found to be more stable, and a large scale array of traps can be realized as shown in Figure 3. The pulsed nDEP helps to reduce the particleparticle interaction. The number of particles trapped in the experiments is close to that predicted by simulation. The discrepancy is probably due to the uncertainty of fabricated channel height. Also, near-wall stick-slip effects and lift-forces may affect the experimental observations.

\section{CONCLUSIONS}

A microfluidic cell-trap array was developed based on the interplay of two set of forces: fluid flow and nDEP. The number of beads trapped is controlled by the voltage and flow rate applied. Pulsing the nDEP force helps to eliminate the particleparticle interaction resulting in a more stable trap. Experimental results obtained with $5 \mu \mathrm{m}$ polystyrene beads are close to that predicted by a simulation model.

\section{ACKNOWLEDGEMENTS}

This work is sponsored by the Singapre-MIT Alliance (SMA) Computational Engineering Program flagship research project.

\section{REFERENCES}

[1] Dino Di Carlo, Liz Y. Wu and Luke P. Lee, Dynamic single cell culture array, Lab on a Chip, 6, 1445, (2006)

[2] Taff, B. M., S. P. Desai, et al., Dielectrophoretically switchable microfluidic weir structures for exclusion-based single-cell manipulation, Micro Total Analysis Systems '07, 8-10, (2007) 